\title{
Signaling Pathways in Trypanosoma cruzi that Modulate Host Cell Interaction
}

\author{
Maria Júlia Manso Alves* and Walter Colli
}

Departamento de Bioquímica, Instituto de Química, Universidade de São Paulo, Brazil

\begin{abstract}
Members of the Gp85/trans-sialidase (Gp85/TS) superfamily and mucins play an essential role in the invasion of host cells by $T$. cruzi trypomastigotes. Together, they constitute a large portion of the genome; approximately 700 and 433 genes encode Gp85/TS glycoproteins and mucins (as do a similar amount of pseudogenes), respectively. Gp85/TS proteins bind to a variety of host cell receptors and extracellular matrix components and binding of TS to host cells is independent of their enzymatic activity. Because mucins are the main substrate for TS, their interaction with host cells has been described as carbohydrate-dependent. Complex signaling cascades operate during the infection process within both parasite and host cells, but most research into signaling events has been limited to those of host cells. Much less information about the parasitic side is available; these pathways will be the subjects of intense research in the near future. Analyses of protein kinases and phosphatases in the parasite genome show pathways common to other organisms, but also parasite-specific pathways that should be exploited as candidates for drug targeting.
\end{abstract}

Keywords: Trypanosoma, kinases, phosphatases, invasion, signaling pathways.

\section{INTRODUCTION}

Trypanosoma cruzi, the etiological agent of Chagas disease, exhibits biological, immunological and pathological diversity. Since the initial identification of the parasite and description of the disease by Carlos Chagas in 1909 [1], a large spectrum of clinical manifestations have been described, ranging from cases that are asymptomatic to those with severe cardiac or digestive complications. Efforts have been made to correlate clinical manifestations with distinct biological $T$. cruzi strains and different parameters have been proposed for classifying the parasite. Recently, two groups, T. cruzi I and II, (with 5 sublineages in group II), associated with the sylvatic and domestic cycles of the parasite, respectively, were identified. However, an analysis of a larger number of samples has pointed to the need for further revision [2]. Clear associations between biological and pathological parameters and genetic classification have not yet been determined. Drawing these types of correlations for a protozoan like $T$. cruzi (which reproduces clonally rather than sexually) has proven to be complicated.

The life cycle of $T$. cruzi includes vertebrate and invertebrate hosts (reduviid insects such as Triatoma infestans, Rhodnius prolixus, Panstrongilus megistus), with an obligatory intracellular stage in the vertebrate. The trypomastigote is the classical non-replicating infective form; metacyclic trypomastigotes (MT) are found in the invertebrate host, and blood trypomastigotes are present in the mammalian host. More than a hundred species of mammals are infected by $T$. cruzi. In humans, an acute phase that may last for 2 months is followed by a long chronic phase, which is asymptomatic in the majority of cases. After several years, $20-35 \%$ of the

*Address correspondence to this author at the Departamento de Bioquímica, Instituto de Química, Universidade de São Paulo Av. Prof. Lineu Prestes 748, 05508-900. São Paulo, Brazil; Tel: (11) 30913810/ ext 231; Fax: (11)30912186; E-mail: mjmalves@iq.usp.br infected individuals will develop irreversible lesions in the heart, intestine or peripheral nervous system [3].

Whereas the basis for this manifestational diversity is not clear, both the host genetic background $[4,5]$ and the capacity of the parasite to deal with the host immune system contribute to the disease outcome. In a typical infection, macrophages are one of the first cells to be infected following phagocytosis of the parasite. Some of the trypomastigotes survive the oxidative burst, in contrast to engulfed epimastigotes, which are normally killed. Interestingly, during the differentiation process of epimastigotes into trypomastigotes, several antioxidant defense proteins are up-regulated [6]. In addition, when 10 strains of $T$. cruzi were compared, a good correlation was found between antioxidant enzyme contents and parasitemia levels; apparently, high antioxidant levels may contribute to the virulence of the parasite [7].

In order to establish a successful infection, trypomastigotes must adhere to the host cell surface and trigger events required for cell entry. These steps are followed by the formation of the parasitophorous vacuole and the escape of the parasite to the cytoplasm of the host cell. Two mechanisms, either dependent or independent on membrane-recruited lysosomes, have been described [8,9]. In both, fusion of the lysosomes with the parasitophorous vacuole is essential. The observation that some trypomastigotes can cross host cells without establishing an infection adds a new level of complexity to the biology of the invasion process [10].

After a variable period of time, trypomastigotes escape to the cytoplasm and differentiate to amastigotes. The desialylation of lysosome membrane glycoproteins by the enzyme trans-sialidase, a member of the gp85/TS gene superfamily is important for this escape because it facilitates pore formation by the trypomastigote-secreted protein Tc-Tox [11]. Amastigotes in the cytoplasm divide for the next 4-5 days and then differentiate back into trypomastigotes, passing through a 
transient [12], proline-dependent, intracellular epimastigote form [13]. It should be mentioned that intracellular forms of T. cruzi have a capacity to transport proline or glucose that fluctuates with the intracellular levels of the metabolite. Amastigotes are the exception; they possess the highest amount of proline despite a barely detectable level of glucose or proline transport [14], which suggests that endogenous protein is the source of the amino acid. Certainly, the metabolic condition of the host cell is important for parasite survival, as the parasite, a purine auxotroph, is unable to synthesize amino acids such as leucine, isoleucine and valine [15]. Although the host cell nucleus does not seem to play an essential role in parasite development and differentiation [16], the necessity of soluble factors secreted by infected or non-infected neighbor cells cannot be ruled out. In fact, a lower number of trypomastigotes was obtained in the enucleated cells when compared to the nucleated cells, reflecting a less favorable environment for nutrient acquisition. Gene expression in the host cell, including metabolic and signaling genes in fibroblast, endothelial and smooth muscle cells, is altered 24 hours post-infection by $T$. cruzi. Interestingly, down-regulation of genes involved in mitotic cell cycle and cell division was also observed, suggesting that infection by T. cruzi may impede host cell cycle progression at late [17], but not early, stages of infection (see $T$. cruzi cell movie at www.sbpz.com.br).

\section{ROLE OF THE T. CRUZI LARGEST GENE FAMI- LIES IN HOST CELL INVASION}

Host cell invasion by $T$. cruzi is the result of multiple molecular interactions, with the consequent activation of a number of signaling pathways, probably involving redundant mechanisms. The invasion mechanisms of extracellular amastigotes appear to differ from those of trypomastigotes and have been a subject of recent review [18]. Therefore, only trypomastigotes will be focused herein.

The role of calcium in the process of $T$. cruzi invasion is supported by data from different laboratories, with an increase in intracellular free calcium concentration detected in the parasite [19] and host cells within $200 \mathrm{~s}$ of interaction with trypomastigotes; a reduction in host cell calcium is correlated with a decrease in the number of parasites in the infected cells [8, 19]. Calcium is essential for the recruitment and fusion of lysosomes with the plasma membrane; it is also required for the rearrangement of actin microfilaments that may facilitate $T$. cruzi invasion by one of several proposed mechanisms [8, 20-23]. The other mechanism of entry, which is lysosome- and actin-independent, occurs with invagination of the host cell plasma membrane [22] and involves the PI3K signaling pathway. Trypomastigote membranes and, to a lesser extent, material constitutively released by trypomastigotes also induced intracellular calcium transients in the host cell. Membrane vesicles shed by trypomastigotes [24] somehow prime host cells for invasion, as they are able to enhance parasite entry into epithelial cells or macrophages and increase the number of amastigote nests in heart tissues [25]. Other molecules also enhance invasion of host cells, such as the glycoprotein Gp83 (released into the matrix by the action of glycosylphosphatidylinositolphospholipase $\mathrm{C}$ following activation of the macrophage MAP kinase pathway) and PKC [23], or members of the
Gp85/trans-sialidase (Gp85/TS) superfamily described below. It should be mentioned that Gp85/TS proteins are released in soluble or membrane-bound forms (vesicles) [24].

Many of the important studies characterizing the interaction of $T$. cruzi trypomastigotes with host cell receptors including bradykinin, thromboxane and protease receptors have recently been reviewed [23]. Only some of them will be highlighted here.

\subsection{Gp85/Trans-Sialidase (Gp85/TS)}

The Gp85/trans-sialidase (Gp85/TS) superfamily of GPIanchored glycoproteins that are present on the parasite surface is subdivided into groups with and without transsialidase activity [26, 27]. Gp85/TS is the largest gene family described in the $T$. cruzi genome (CL Brener strain), constituted by more than 700 genes and almost 700 pseudogenes [28]. Approximately 140 genes encode trans-sialidase proteins. Of these, 70 genes code for enzymatically active proteins and the other half for inactive forms. These proteins are inactive owing to the mutation of a key tyrosine residue, but they still bind sialic acid and galactose substrates [27, 29].

TS proteins are capable of transferring sialic acid from host glycoconjugates to parasite macromolecules [27, 29, 30], such as the mucins that are abundant on the surface of $T$. cruzi. Enzymatic activity does not seem to be important for the invasion step; both active and inactive TS bind to a variety of host cells, mediating parasite entry [21]. Some forms are expressed only in trypomastigotes $[21,30]$; the relationship between the levels of a few highly expressed TS members and infectivity of the parasite has been established [21, 23, 30]; sialylation of $T$. cruzi surface molecules confers resistance to complement [31]; sialylated compounds interact with the inhibitory sialic acid-binding protein Siglec-E (sialic acid-binding Ig-like lectin-E) predominantly expressed on cells of the immune system, inhibiting the activation of immune cells [32] TS induces apoptosis in cells of the immune system due to acquisition of sialic acid [33].

Tc85 proteins, a subgroup of Gp85/TS, are mainly expressed in tissue-cultured trypomastigotes (TCT); an equivalent GP82 was described in metacyclic trypomastigotes. Both are devoid of trans-sialidase activity and are transcribed in infective and non-infective stages. Expression of GP82 and Tc85, as described for other genes in trypanosomatids $[34,35]$ is regulated by post-transcriptional mechanisms [36, 37]. In fact, the mobilization of GP82 mRNA to the polysomes is positively or negatively regulated by proteins whose levels can themselves be mediated by an element present in the $3^{\prime}$-UTR of the transcripts [37].

At least some proteins behave as multi-adhesion molecules, as has been shown for Tc85-11, a cloned member of the Tc85 subset, which binds to laminin by the N-terminal domain $[38,39]$ and to cytokeratin-18 by the carboxyl subterminal VTV motif of the Gp85/TS superfamily (herein called FLY domain) [40]. FLY also binds to other uncharacterized molecules on both epithelial and non-epithelial cells [41]. A FLY-containing synthetic peptide activates the ERK1/2 signaling pathway, which Gp85/TS proteins participate in $[42,43]$ and also induces cytoskeleton rearrangement in the host cell, enhancing invasion by $T$. cruzi [41]. Upregulation of $T$. cruzi entry into endothelial cells through a 
FLY-independent region has also been described for one inactive TS protein, but the specific sequence involved was not identified [42].

In addition to participating in the invasion of host cells, non-catalytic regions of TS exert other effects, for example the activation of survival signal pathways including MAPK and PI3K/Akt signaling in neurons, glial, dendritic or epithelial cells [43-46]. TS binds to sialic acid-containing molecules on the surface of endothelial cells leading to NF- $\mathrm{KB}$ activation, expression of adhesion molecules (ICAM-1, VCAM-1 and E-selectin) and blockage of cell apoptosis normally induced by growth factor deprivation [42].

Gp82 is the principal member of the Gp85/TS superfamily involved in MT invasion of host cells, and its expression has been correlated with invasion capacity [31]. Gp82 binds to gastric mucin and seems to be an important element in infection by the oral route [31], wherein food contaminated with the insect stages of $T$. cruzi is ingested. This manner of transmission is responsible for the occurrence of recent microepidemics in Brazil and other American countries [47]. Gp82 triggers calcium mobilization in the host cell and $\mathrm{Ca}^{2+}$-dependent cytoskeletal disorganization, which facilitates parasite invasion [31]. In MT, phosphorylation of a $175-\mathrm{kDa}$ protein by a protein kinase activated during parasite invasion, and the generation of inositol 1, 4, 5-triphosphate (IP3) by phospholipase $\mathrm{C}$ (which promotes calcium release from IP3-sensitive stores), have been described [31]. Metacyclic trypomastigotes from distinct strains express different amounts of GP82, GP30 and GP90. It is claimed that GP82 (and GP 30) is the principal actor in the invasion process. Since strains that express high levels of GP82 and GP90 are poor invaders, the latter was proposed to be a negative modulator of the invasion. Eventually, the strain expressing high levels of GP90 would become infective provided that this protein is digested in the intestinal tract, thus explaining the relative effectiveness of the oral infection route [31].

\subsection{Mucins}

The second largest family of genes in the parasite encodes $T$. cruzi mucins, which are also GPI-anchored surface glycoproteins. Approximately $4 \times 10^{6}$ mucins cover the parasite surface [26-29]. The small core polypeptide (50 to 200 amino acids length) is rich in carbohydrates O-linked to serine and threonine [see 27, 29, 48] for a detailed review on the structure and function of mucins]. In the insect-derived stages (epimastigotes and MT), mucins have a more homogeneous polypeptide composition, showing a double or triple band in the 35 to $50 \mathrm{kDa}$ range in electrophoretic separations, as compared to TCT mucins, which on PAGE reveal as a smear from 60 to $200 \mathrm{kDa}$. TCT - and other intermediate mammalian stages - express a third group of mucins, called small surface antigens [29], which increase the mucin heterogeneity present in trypomastigotes.

Other stage-dependent differences in mucin structure have described, such as the presence in trypomastigote mucins of the epitope Gal $(\alpha-1,3) \mathrm{Gal}$, the main target of antibodies response in Chagasic patients [49], or the lipid composition of the GPI-anchor that induces a proinflammatory response via MAPK and NF- $\mathrm{KB}$ cascades through activation of Toll-receptors [50].
In addition to the protective role of $T$. cruzi mucins play against the proteases of the insect digestive tract, they have been implicated in attachment to, and invasion of, the host. Galactofuranose, which mainly decorates oligosaccharide chains in mucins from epimastigotes [27, 48], is responsible for adhesion of epimastigotes to the intestinal tract. This adhesion step is implicated in the differentiation to MT, as has been shown for T. cruzi GIPL-1, a galactofuranose-rich glycophosphoceramide [51]. Several pieces of evidence support a role for carbohydrates such as mannose or galactose in the invasion of $T$. cruzi [23]. Carbohydrates from the $35-50 \mathrm{kDa}$ mucins are important for invasion of various cell types [52, 53] and removal of sialic acid from the surface of the parasite by sialidase treatment enhances the invasion capacity of T. cruzi [54]. In addition, mucins from trypomastigotes trigger calcium mobilization, an essential step in the invasion mechanism.

It has been proposed that galectin-3 establishes a bridge between mucins and laminin, thus recruiting trypomastigotes to the extracellular matrix and increasing adhesion of trypomastigotes to cells. Interestingly, galectin-3 expression is upregulated during infection [55].

GPI-mucin-like proteins from trypomastigotes, but not from epimastigotes, trigger MAPK and NF- $\mathrm{KB}$ cascades through Toll-like receptor activation $[49,50]$ and are therefore responsible for the recognition of microbial and viral molecules and the induction of tolerance by macrophages.

\section{T. CRUZI AND INTERACTION WITH EXTRACEL- LULAR MATRIX}

Binding of trypomastigotes to elements of the extracellular matrix such as laminin, fibronectin, heparan sulphate, thrombospondin, galectin-3 or collagen IV is partially, but not exclusively, achieved by mucins and members of the Gp85/TS family. In most cases, the $T$. cruzi ligand was not characterized $[21,56]$. The interaction of a trypomastigote $85-\mathrm{kDa}$ surface protein with the RGD sequence of fibronectin was one of the first descriptions of the binding of T. cruzi to extracellular elements [57]. The role of thrombospondin A and galectin-3 [58, 59], as well as laminin $\gamma 1$ [60], in the invasion by $T$. cruzi was shown using RNAi, confirming previous observations [38]. Binding of T. cruzi to heparan sulfate proteoglycans through a $60-\mathrm{kDa}$ heparinbinding protein known as penetrin $[61,62]$ was characterized as $\mathrm{N}$-sulfation and glucuronic acid-dependent; heparin inhibited the invasion of cardiomyocytes by $T$. cruzi by $84 \%$ [63]. To our knowledge, neither the 60-kDa protein nor its signaling pathway was further characterized. It should be kept in mind that sequential changes to connective matrix components lead not only to parasite binding, but also to the heart tissue fibrosis characteristic of Chagas disease [55, 64, 65]. In vitro studies focusing on the remodeling of extracellular matrix proteins have had contradictory results, apparently dependent on experimental conditions [66, 67].

Transforming growth factor $\beta$ (TGF- $\beta$ ) has been implicated in stimulation of fibrosis and parasitic cellular invasion and growth in Chagas disease, as well as in host immunity down-regulation [68]. Interestingly, the parasite is able to capture TGF- $\beta$ from the host, an essential step for the differentiation of amastigotes to trypomastigotes [69]. The interac- 
tion of TGF- $\beta$ with its receptors on the host cell membrane leads to the phosphorylation of Smad-2 and Smad-3 in the cytoplasm, which subsequently form a complex with Smad4. This complex translocates to the nucleus, inducing transcription of different genes such as those encoding for fibronectin, thrombospondin and collagen I, and probably contributing to heart fibrosis. Interestingly, a small inhibitor of TGF- $\beta$ signaling (SB-431542) administered to experimental animals in the acute phase of infection was a potent inhibitor of parasitemia and heart injury and is being proposed for clinical use [70-72].

\section{SIGNALING PATHWAYS IN T. CRUZI DURING HOST CELL INVASION}

Until recently, knowledge of the signaling pathways activated during invasion by $T$. cruzi has been limited to those of the host cell. This scenario should change rapidly due to genome sequencing of trypanosomatids. In the particular case of the T. cruzi genome, genes encoding 190 protein kinases (19 being atypical protein kinases) and 86 phosphatases [72, 73] have been identified.

A detailed comparative analysis of the kinomes of $T$. cruzi, T. brucei and L. major showed the presence of a group of proteins involved in numerous pathways well established in other organisms, as well as key differences, as the lack of tyrosine kinases and tyrosine kinase-like groups in trypanosomatids. However, because protein tyrosine phosphorylation is described as a common modification of parasite proteins, activity of atypical tyrosine kinases or dual-specificity kinases have been proposed. Absence of common accessory domains present on human protein kinases (Ig, fn3, SH2 and $\mathrm{SH} 3$ ) or the lack of guanylyl cyclase receptor proteins in trypanosomatids are examples of other differences pointed out when trypanosomatids and human genomes are compared, regarding protein kinases.

The majority of the phosphatases in $T$. cruzi belong to the serine/threonine family; the parasite has a lower proportion of tyrosine phosphatases than other eukaryotic genomes [73]. This reduced number of tyrosine phosphatases is consistent with the lack of tyrosine kinases also observed.

Extensive reviews on protozoan MAP kinases [74], PI3kinases [75] protein serine/threonine phosphatases [76], tyrosine phosphatases [77] and cyclic nucleotide signaling mechanisms [78] have been published recently. It should be pointed out that in contrast to the large number of kinases and phosphatases, only a restricted number of GTPase families are present in trypanosomes, with a complete absence of the heterotrimeric GTPases that are key elements in the signaling processes of other eukaryotes. So far, little is known about the contribution of small GTPases [79].

It is interesting to consider the idea that the flagellum may act as a sensory organ in the MAP kinase pathway [80]. This suggestion was based on the observations that various kinases are involved in the control of the flagellum length, and that a sensory function has been attributed to cilia and flagella in other organisms. Glycosomes and acidocalcisomes, which play important role in energy metabolism in trypanosomatids, are involved in the cellular responses triggered by nutrients. Differentiation in T. brucei is regulated by a phosphatase cascade, with the localization of a key unusual type of Ser/Thr phosphatase in the glycosome [81]. The role of acidocalcisomes is coupled to TOR (the target of rapamycin), an evolutionarily conserved atypical protein kinase that belongs to the phosphoinositide 3-kinase-related kinase family. In mammals, TOR signal pathway integrates intracellular and extracellular signals and is activated during various cellular processes [82]. TOR3, one of the three TOR kinases identified in Leishmania major is involved in the acidocalcisome biogenesis and infectivity, since Tor3 $3^{-}$null mutants are unable to survive in macrophages and infectivity to mice is highly attenuated [83]. Two TOR kinases control cell growth in Trypanosoma brucei [84] and one of the two unusual TOR-like kinases identified, the cytosolic protein TOR-like 1 kinase, is involved in polyphosphate level and acidocalcisome maintenance [85].

Despite the annotation of a great number of kinases and phosphatases in the genome, very little is known about their role in $T$. cruzi signaling during invasion. One of the few examples is the phosphorylation of a $175-\mathrm{kDa}$ protein during the invasion of MT into host cells by a protein kinase, as pointed out above. Recently, it was shown that treating $T$. cruzi metacyclic or tissue culture trypomastigotes with inhibitors or with specific anti-sense oligonucleotides of calcineurin B inhibits parasite invasion. Although no elucidation of the enzyme-mediated signaling pathways has yet been achieved, this observation points to the relevance of phosphatases in parasite internalization [86]. Interestingly, the parasitic enzyme, in contrast to its human counterpart, lacks calmodulin-binding and auto-inhibitory domains [87].

\section{CONCLUDING REMARKS}

A plethora of molecules that pass between the parasite and the host are responsible for the establishment of infection. Of these, molecules involved in the adhesion step and the ensuing signaling pathways are particularly being considered as possible drug-target candidates. In addition to more classical approaches, the SELEX method (Systematic Evolution of Ligands by Exponential Enrichment), an oligonucleotide-based combinatorial library approach can be used to isolate high-affinity ligands (aptamers) for a wide variety of molecules, as was done for proteins from the extracellular matrix that interact with $T$. cruzi $[88]$ and could be used as potential targets.

The genomic sequences of Trypanosoma and Leishmania species reveal protein kinases and phosphatases with peculiar characteristics, such as a paucity or absence of tyrosine kinases and phosphatases, or of the accessory domains typically responsible for protein-protein interaction $(\mathrm{IgG}, \mathrm{fn} 3$, $\mathrm{SH} 3$ or SH2 domains) in mammalian signaling pathways. As in other human diseases, differences like these between parasitic and mammalian signaling point to good targets for drug discovery. In addition to the target-base approach employed for drug discovery, the high-throughput drug screening can provide new chemical structures on which to base an effective compound. Interestingly, the majority of 4,205 compounds selected from the almost 2 million tested against asexual blood stage of $P$. falciparum, might target kinases of the parasite [89]. If they are directed to a small number or to a large spectrum of kinases is, as yet, unknown. Although the criteria to select a good inhibitor of kinases or phosphatases is not under discussion here, it should be recalled that 
specific modulation of enzymes or protein-protein interactions by small peptides is one possibility. Small peptides bind more specifically, can target more than one interaction site, are effective at lower concentrations and can be delivered as prodrugs by coupling to other molecules. However, rapid turnover due to low stability and high susceptibility to intracellular protease degradation may be disadvantageous, as pointed out in a recent review [90].

In summary, the differences in signal cascades between the parasite and the host ought to be exploited as a probable locale for drug targeting.

\section{ACKNOWLEDGEMENTS}

This work was supported in part by the Fundação de Amparo à Pesquisa do Estado de São Paulo (FAPESP) and Conselho Nacional de Desenvolvimento Científico e Tecnológico $(\mathrm{CNPq})$

Due to space limitations, recent reviews are cited preferentially over the original research papers.

\section{REFERENCES}

[1] Chagas C. Nova tripanozomiaze humana. Mem Inst Oswaldo Cruz 1909; 1:11-72.

[2] Zingales B, Andrade SG, Briones MRS, et al. A new consensus for Trypanosoma cruzi intraspecific nomenclature: second revision meeting recommends Tc I to Tc VI. Mem Inst Oswaldo Cruz 2009; 104:1051-54.

[3] Moncayo A, Silveira AC. Current epidemiological trends for Chagas disease in Latin America and future challenges in epidemiology, surveillance and health policy. Mem Inst Oswaldo Cruz 2009; 104: 17-30.

[4] Williams-Blangero S, VandeBerg JL, Blangero J, Correa-Oliveira $\mathrm{R}$. Genetic epidemiology of Trypanosoma cruzi infection and Chagas' disease. Front Biosci 2003; 8: 337-45.

[5] Marinho CRF, Bucci DZ, Dagli MLZ, et al. Pathology affects different organs in two mouse strains chronically infected by a Trypanosoma cruzi clone: a model for genetic studies of Chagas'disease. Infect Immun 2004; 72: 2350-57.

[6] Piacenza L, Alvarez MN, Peluffo G, Radi R. Fighting the oxidative assault: the Trypanosoma cruzi journey to infection. Cur Opin Microbiol 2009; 12: 415-21.

[7] Piacenza L, Zago MP, Peluffo G, Alvarez MN, Basombrio MA, Radi R. Enzymes of the antioxidant network as novel determiners of Trypanosoma cruzi virulence. Int J Parasitol 2009; 39: 1455-64.

[8] Andrade LO, Andrews NW. The Trypanosoma cruzi host cell interplay: location, invasion and retention. Nat Rev Microbiol 2005; 3: 819-23

[9] Burleigh BA. Host cell signaling in Trypanosoma cruzi invasion: do all roads lead to lysosomes? Sci STEK 2005; 293: p. 36.

[10] Schenkman S, Robbins ES, Nussenzweig V. Attachment of Trypanosoma cruzi to mammalian cells requires parasite energy, and invasion can be independent of the target cell cytoskeleton. Infect Immun 1991; 59: 645-54.

[11] Hall BF, Webster P, Ka MA, Joiner KA, Andrews NW. Desialylation of lysosomal membrane glycoprotein by Trypanosoma cruzi: a role for the surface neuraminidase in facilitating parasite entry into the host cell cytoplasm. J Exp Med 1992; 176: 313-25.

[12] Almeida-de-Faria M, Freymuller E, Colli W, Alves MJM. Trypanosoma cruzi: Characterization of an intracellular epimastigotelike form. Exp Parasitol 1999; 92: 263-74.

[13] Tonelli RR, Silber AM, Almeida-de-Faria M, Hirata IY, Colli W, Alves MJM. L-Proline is essential for the intracellular differentiation of Trypanosoma cruzi. Cell Microbiol 2004; 6: 733-41.

[14] Silber AM, Tonelli RR, Lopes CG, et al. Glucose uptake in the mammalian stages of Trypanosoma cruzi. Mol Biochem Parasitol 2009; 168: 102-08.

[15] Silber AM, Colli W, Ulrich H, Alves MJM, Pereira CA. Amino acid metabolic routes in Trypanosoma cruzi: possible therapeutic targets against Chagas'disease. Curr Drugs Targets Infect Disord 2005; 5: 53-64.
[16] Coimbra VC, Yamamoto D, Khusal KG, et al. Enucleated L929 cells support invasion, differentiation, and multiplication of Trypanosoma cruzi parasites. Infect Immun 2007; 75: 3700-06.

[17] Costales JA, Daily JP, Burleigh BA. Cytokine-dependent and independent gene expression changes and cell cycle block revealed in Trypanosoma cruzi-infected host cells by comparative mRNA profiling. BMC Genomics 2009; 10: 252-69.

[18] Mortara RA, Andreoli WK, Fernandes MC, et al. Host cell actin remodeling response to Trypanosoma cruzi: trypomastigote versus amastigote entry. Subcell Biochem 2008; 47: 101-09.

[19] Moreno SNJ, Silva J, Vercesi AE. Cytosolic-free calcium elevation in Trypanosoma cruzi is required for cell invasion. J Exp Med 1994; 180: 1535-40.

[20] Burleigh BA, Woolsey AM. Cell signaling and Trypanosoma cruzi invasion. Cell Microbiol 2002; 4: 701-11.

[21] Alves MJM, Colli W. Trypanosoma cruzi: adhesion to the host cell and intracellular survival. IUMB Life 2007; 59: 274-79.

[22] Alves MJM, Mortara R. A century of research: what we learned about the interaction of Trypanosoma cruzi with host cells? Mem Inst Oswaldo Cruz 2009; 104: 76-88.

[23] Villalta F, Scharfstein J, Ashton AW, et al. Perspectives on the Trypanosoma cruzi-host cell receptor interactions. Parasitol Res 2009; 104: 1251-60.

[24] Gonçalves MF, Umezawa ES, Katzin AM, et al. Trypanosoma cruzi: shedding of surface antigens as membrane vesicles. Exp Parasitol 1991; 72: 43-53.

[25] Torrecilhas ACT, Tonelli RR, Pavanelli WR, et al. Trypanosoma cruzi: parasite shed vesicles increase heart parasitism and generate intense inflammatory response. Microbes Infect 2009; 11: 29-39.

[26] Frasch AC. Functional diversity in the trans-sialidase and mucin families in Trypanosoma cruzi. Parasitol Today 2000; 16: 282-86.

[27] De Lederkeremer RM, Agusti R. Glycobiology of Trypanosoma cruzi. Adv Carbohydr Chem Biochem 2009; 62: 311-66.

[28] El-Sayed NM, Myler PJ, Bartholomeu DC, et al. The genome sequence of Trypanosoma cruzi, etiologic agent of Chagas disease. Science 2005; 309: 409-15.

[29] Buscaglia CA, Campo VA, Frasch ACC, di Noia JM. Trypanosoma surface mucins: host dependent coat diversity. Nat Rev Microbiol 2006; 4: 229-36.

[30] Schenkman S, Eichinger D, Pereira MEA, Nussensweig V. Structural and functional properties of trypanosome trans-sialidase. Ann Rev Microbiol 1994; 48: 499-532.

[31] Yoshida N. Trypanosoma cruzi infection by oral route. How the interplay between parasite and host components modulates infectivity. Parasitol Int 2008; 57: 105-09.

[32] Jacobs T, Erdmann H, Fleischer B. Molecular interaction of Siglecs (sialic acid-binding Ig-like lectins) with sialylated ligands on Trypanosoma cruzi. Eur J Cell Biol 2010; 89: 113-16.

[33] Mucci J, Risso MG, Leguizamón MS, Frasch ACC, Campetella O. The trans-sialidase from Trypanosoma cruzi triggers apoptosis by target cell sialylation. Cell Microbiol 2006; 8: 1086-95.

[34] Palenchar JB, Bellofatto V. Gene transcription in trypanosomes. Mol Biochem Parasitol 2006; 146: 135-41.

[35] Noé G, de Gaudenzi JG, Frasch AC. Functionally related transcripts have common RNA motifs for specific RNA-binding proteins in trypanosomes. BMC Mol Biol 2008; 9: 107.

[36] Abuin G, Freitas-Junior LH, Colli W, Alves MJM, Schenkman S. Expression of trans-sialidase and $85 \mathrm{kDa}$ glycoprotein genes in Trypanosoma cruzi is differentially regulated at the posttranscriptional level by labile protein factors. J Biol Chem 1999; 274: 13041-47.

[37] Gentil LG, Cordero EM, do Carmo MS, dos Santos MRM, da Silveira JF. Posttranscriptional mechanisms involved in the control of expression of the stage-specific GP82 surface glycoprotein in Trypanosoma cruzi. Acta Trop 2009; 109: 152-58.

[38] Giordano R, Fouts D, Tewari D, Colli W, Manning J, Alves MJM Cloning of a surface membrane glycoprotein specific for the infective form of Trypanosoma cruzi having adhesive properties to laminin. J Biol Chem 1999; 274: 3461-68.

[39] Marroquin-Quelopana M, Oyama S, Pertinhez TA, et al. Modeling the Trypanosoma cruzi Tc85-11 protein mapping the lamininbinding site. Biochem Biophys Res Commun 2004; 325: 612-18.

[40] Magdesian MH, Giordano R, Ulrich $\mathrm{H}$, et al. Infection by Trypanosoma cruzi: identification of a parasite ligand and its host- cell receptor. J Biol Chem 2001; 276: 19382-89. 
[41] Magdesian MH, Tonelli RR, Fessel MR, et al. A conserved domain of the gp $85 /$ trans-sialidase family activates host cell extracellular signal- regulated kinase and facilitates Trypanosoma cruzi infection. Exp Cell Res 2007; 313: 210-18.

[42] Dias WB, Fajardo FD, Graça-Souza AV, et al. Endothelial cell signaling induced by trans-sialidase from Trypanosoma cruzi. Cell Microbiol 2008; 10: 88-99.

[43] Chuenkova MV, Pereira M. A trypanosomal protein synergizes with the cytokines ciliary neurotrophic factor and leukemia inhibitory factor to prevent apoptosis of neuronal cells. Mol Biol Cell 2000; 11:1487-98.

[44] Chuenkova MV, Furnari FB, Cavenee WK, Pereira M. Trypanosoma cruzi trans-sialidase: a potent and specific survival factor for human Schwann cells by means of phosphatidylinositol 3kinase/Akt signaling. Proc Natl Acad Sci USA 2001; 98: 9936-41.

[45] Chuenkova MV, PereiraPerrin M. A synthetic peptide modeled on PDNF, Chagas'disease parasite neutrophic factor, promotes survival and differentiation of neuronal cells through TrkA receptor. Biochemistry 2005; 44: 15685-94

[46] Melo-Jorge M, PereiraPerrin M. The Chagas'disease parasite Trypanosoma cruzi exploits nerve growth factor receptor TrkA to infect mammalian hosts. Cell Host Microbe 2007; 1: 251-61.

[47] Coura JR. Transmission of chagasic infection by oral route in the natural history of Chagas'disease. Rev Soc Bras Med Trop 2006; 39: 113-17.

[48] Mendonça-Previato L, Todeschini AR, Heise N, Previato JO. Protozoan parasite-specific carbohydrate structures. Curr Opin Struct Biol 2005; 15: 499-05.

[49] Ropert C, Ferreira LR, Campos MA, et al. Macrophage signaling by glycosylphosphatidylinositol-anchored mucin-like glycoproteins derived from Trypanosoma cruzi trypomastigotes. Microbes Infect 2002; 4: 41015-25.

[50] Campos MA, Gazzinelli RT. Trypanosoma cruzi and its components as exogenous mediators of inflammation recognized through Toll-like receptors. Mediators Inflamm 2004; 13: 139-43.

[51] Nogueira NFS, Gonzalez MS, Gomes JE, et al. Trypanosoma cruzi: involvement of glycoinositolsphospholipids in the attachment to the luminal midgut surface of Rhodnius prolixus. Exp Parasitol 2007; 116: 120-28.

[52] Ruiz RC, Favoreto S, Dorta ML, Oshiro MEM, Ferreira AT, Manque PM. Infectivity of Trypanosoma cruzi strains is associated with differential expression of surface glycoproteins with differential $\mathrm{Ca}^{+}$signaling activity. Biochem J 1998; 330: 505-11.

[53] Turner CW, Lima MF, Villalta F. Trypanosoma cruzi uses a 45$\mathrm{kDa}$ mucin for adhesion to mammalian cells. Biochem Biophys Res Commun 2002; 290: 29-34

[54] Yoshida N, Dorta ML, Ferreira AT, et al. Removal of sialic acid from mucin-like surface molecules of Trypanosoma cruzi metacyclic trypomastigotes enhances parasite-host cell interaction. Mol Biochem Parasitol 1997; 84: 57-67.

[55] Vray B, Camby I, Vercruysse V, et al. Up-regulation of galectin-3 and its ligands by Trypanosoma cruzi infection with modulation of adhesion and migration of murine dendritic cells. Glycobiology 2004; 14: 647-57.

[56] Ulrich H, Magdesian MH, Alves MJM, Colli W. In vitro selection of RNA aptamers that bind to cell adhesion receptors of Trypanosoma cruzi and inhibit cell invasion. J Biol Chem 2002; 277: 20756-62.

[57] Ouaissi MA, Afchain D, Capron. Fibronectin receptors on Trypanosoma cruzi trypomastigotes and their biological function. Nature $1984 ; 308: 380-2$

[58] Simmons KJ, Nude PN, Kleschchenko YY, Lima MF, Villalta F. Stable RNA interference of host thrombospondin-1 blocks Trypanosoma infection. FEBS Lett 2006; 580: 2365-70.

[59] Moody T, Ochieng J, Villalta F. Novel mechanism that Trypanosoma cruzi uses to adhere to the extracellular matrix-mediated by human galectin-3. FEBS Lett 2000; 470: 305-08.

[60] Nde PN, Simmons KJ, Kleshchenko YY, Pratap S, Lima MF, Villalta F. Silencing of the laminin gamma-1 gene blocks Trypanosoma cruzi infection. Infect Immun 2006; 74: 1643-8.

[61] Herrera EM, Ming M, Ortega-Barria E, Pereira ME. Mediation of Trypanosoma cruzi invasion by heparan sulfate receptors on host cells and penetrin counterreceptors on the trypanosomes. Mol Biochem Parasitol 1994; 65: 73-83.

[62] Calvet CM, Toma L, de Souza FR, Meirelles MNL, Pereira MCS. Heparan sulfate proteoglycans mediate the invasion of cardiomyo- cytes by Trypanosoma cruzi. J Eukaryot Microbiol 2003; 50: 97103.

[63] Oliveira Jr FOR, Alves CR, Calvet CM, et al. Trypanosoma cruzi heparin-binding proteins and the nature of the host cell heparin sulfate-binding domain. Microb Pathog 2008; 44: 329-38.

[64] Andrade SG, Grimaud JA, Stocker-Guerret S. Sequential change of the connective matrix components of the myocardium (fibronectin and laminin) and evolution of cardiac fibrosis in mice infected with Trypanosoma cruzi. Am J Trop Med Hyg 1989; 40: 252-60.

[65] Pinho RT, Vannier-Santos MA, Alves CR, Marino APMP, Castello Branco LRR, Lannes-Vieira J. Effect of Trypanosoma cruzi released antigens binding to non-infected cells on anti-parasite antibody recognition and expression of extracellular matrix components. Acta Trop 2002; 83: 103-15.

[66] Garzoni LR, Adesse D, Soares MJ, Rossi MID, Borojevic R, Meirelles MNL. Fibrosis and hypertrophy induced by Trypanosoma cruzi in a three-dimensional cardiomyocyte- culture system. $\mathrm{J}$ Infec Dis 2008; 197: 906-15.

[67] Calvet CM, Oliveira Jr FOR, Araujo-Jorge TC, Pereira MCS Regulation of extracellular matrix expression and distribution in Trypanosoma cruzi- infected cardiomyocytes. Int J Med Microbiol 2009; 299: 301-12.

[68] Araújo-Jorge TC, Waghabi MC, Soieiro M, Keramidas M, Bailly S, Feige JJ. Pivotal role of TGF- $\beta$ in infectious heart disease: the case of Trypanosoma cruzi infection and consequent Chagasic myocardiopathy. Cytokine Growth Factor Rev 2008; 19: 405-13.

[69] Waghabi MC, Keramidas M, Bailly S, et al. Uptake of host cell transforming growth factor-beta by Trypanosoma cruzi amastigotes in cardiomyocytes: potential role in parasite cycle completion. Am J Pathol 2005; 167: 993-1003.

[70] Waghabi MC, Keramidas M, Calvet CM, et al. SB-431542, a transforming growth factor beta inhibitor, impairs Trypanosoma cruzi infection in cardiomyocytes and parasite cycle completion. Antimicrob Agents Chemother 2007; 51: 2905-10.

[71] Waghabi MC, de Souza EM, de Oliveira GM, et al. Pharmacological inhibition of transforming growth factor $\beta$ signaling decreases infection and prevents heart damage in acute Chagas'disease. Antimicrob Agents Chemother 2009; 53: 4694-701.

[72] Parsons M, Worthey EA, Ward PN, Mottram JC. Comparative analysis of the kinomes of three pathogenic trypanosomatids: Leishmania major, Trypanosoma brucei and Trypanosoma cruzi. BMC Genomics 2005; 6: 127.

[73] Brenchley R, Tariq H, McElhinney H, et al. The TriTryp phosphatoma: analysis of the protein phosphatase catalytic domains. BMC Genomics 2007; 8: 434.

[74] Wiese M. Leishmania MAP Kinases- Familiar proteins in an unusual context. Int J Parasitol 2007; 37: 1053-62.

[75] Bahia D, Oliveira LM, Lima FM, et al. The TryPIkinome of five human pathogenic trypanosomatids: Trypanosoma brucei, Trypanosoma cruzi, Leishmania major, Leishmania braziliensis and Leishmania infantum- New tools for designing specific inhibitors. Biochem Biophys Res Commun 2009; 390: 963-70.

[76] Kutuzov MA, Andreeva A. Protein Ser/Thr phosphatases of parasitic protozoa. Mol Biochem Parasitol 2008; 161: 81-90.

[77] Andreeva AV, Kutuzov MA. Protozoan protein tyrosine phosphatases. Int J Parasitol 2008; 38: 1279-95.

[78] Laxman S, Beavo JA. Cyclic nucleotide signaling mechanisms in trypanosomes: possible targets for therapeutic agents. Mol Interv 2007; $7: 203-15$.

[79] Field MC, O’Reilly AJ. How complex is GTPase signaling in trypanosomes? Trends Parasitol 2008; 24: 243-57.

[80] Routureau B, Morales MA, Basin P, Spath GF. The flagellummitogen-activated protein kinase connection in Trypanosomatids: a key sensory role in parasite signaling and development? Cell Microbiol 2009; 11: 710-18.

[81] Szoor B, Ruberto I, Burchmore R, Matthews KR. A novel phosphatase cascade regulates differentiation in Trypanosoma brucei via a glycosomal signaling pathway. Genes Dev 2010; 24: 1306-16.

[82] Laplante M, Sabatini DM. mTOR signaling at a glance. J Cell Sci 2009; 122: 3589-94

[83] Madeira da Silva L, Beverley SM. Expansion of the target of rapamycin (TOR) kinase family and function in Leishmania shows that TOR3 is required for acidocalcisome biogenisis and animal infectivity. Proc Natl Acad Sci USA 2010; 29: 11965-70. 
[84] Barquilla A, Crespo JL, Navarro M. Rapamycin inhibits trypanosome cell growth by preventing TOR complex 2 formation. Proc Natl Acad Sci USA 2008; 105: 14579-84.

[85] De Jesus TCL, Tonelli RR, Nardelli SC, et al. Tor-like 1 kinase is involved in the control of polyphosphate levels and acidocalcisome maintenance in Trypanosoma brucei. J Biol Chem 2010; 285: 24131-40.

[86] Araya JE, Cornejo A, Orrego PR, et al. Calcineurin B of the human protozoa parasite Trypanosoma cruzi is involved in cell invasion. Microbe Infect 2008; 10: 892-900.
[87] Moreno VR, Aguero F, Tekiel V, Sanchez DO. The calcineurin A homologue from Trypanosoma cruzi lacks two important regulatory domains. Acta Trop 2007; 101: 80-9.

[88] Ulrich $\mathrm{H}$, Wrenger C. Disease- specific biomarker discovery by aptamers. Cytometry A 2009; 75A: 727-33.

[89] Gamo F-J, Sanz LM, Vidal J, et al. Thousands of chemical starting points for antimalarial lead identification. Nature 2010; 465: 30510 .

[90] Costa-Junior HM, Suetsugu MJ, Krieger JE, Schechtman D. Specific modulation of protein kinase activity via small peptides. Regul Pept 2009; 153: 11-18.

(C) Alves and Colli; Licensee Bentham Open.

This is an open access article licensed under the terms of the Creative Commons Attribution Non-Commercial License (http://creativecommons.org/licenses/by-nc/3.0/) which permits unrestricted, non-commercial use, distribution and reproduction in any medium, provided the work is properly cited. 